(NASA-TM-110507) SIMULATED

RAREFIED ENTRY OF THE GALILEO PROBE

INTO THE ATMOSPHERE OF JUPITER

(NASA. Ames Research (enter) $8 \mathrm{p}$
N95-21621

Unclas

G3/91 0041081 


\section{Simulated Rarefied Entry of the Galileo Probe into the Atmosphere of Jupiter}

\author{
Brian L. Haas* \\ Eloret Institute, Palo Alto, California 94303 \\ Frank S. Milos $\dagger$ \\ NASA Ames Research Center, Moffett Field, California 94035-1000
}
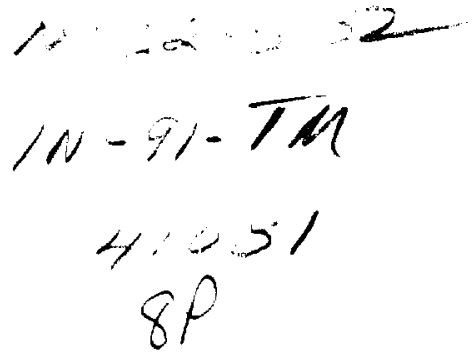

Flow properties and aerodynamics are computed with a direct simulation Monte Carlo (DSMC) method for rarefied entry of the Galileo Probe into the atmosphere of Jupiter. Accurate predictions of vehicle drag coefficients are needed in order to assess atmospheric properties from the onboard Atmosphere Structure Experiment where highly-sensitive accelerometers will measure the drag force to within 10-6 bart during the initial entry phase at high altitudes. The corresponding flow rarefaction extends from the free molecule limit to the near continum transition regime ( $R e<1000$ ). Simulation results indicate that $C_{\mathrm{D}}$ varies from 2.1 at the free molecule limit down to 1.6 at $\mathrm{Re}_{\infty}=1,000$. Temperatures, densities, and internal energies throughout the flow field were also computed at each altitude ranging from $735 \mathrm{~km}$ to $353 \mathrm{~km}$ above the $1 \mathrm{barr}$ level in the Jovian atmosphere. Surface heating and temperatures of the probe were computed directly in the DSMC code by assuming radiative equilibrium. Material response was re-assessed accurately during entry by accounting for conductivity, heat capacity, and pyrolysis which led to surface material mass effux several times that of the freestream mass infiux. The simulation also accounted for the quantum nature of the rotational energy mode of the dominant atmospheric species $\mathrm{H}_{2}$ through partial intemal excitation in the freestream gas.

\section{NOMENCLATURE}

$A$

- Research Scienuish Aerohemodyamies Branch. Menber, AI Mailing Address: NASA Ames Research Center, M/S 230-2 Moffet Field, CA 94035-1000.

† Research Scientist, Thermal Prolection Materials Branch. Moffet Field, CA 94035-1000.

This paper is doclured a wort of the U.S. Government and is na subjoct to copyright protection in the United States.

\section{Subscripts:}

$\begin{array}{ll}\text { D } & \text { deep-space value } \\ \text { ref } & \text { reference value } \\ r & \text { pertains to the rotational mode } \\ v & \text { pertains to the vibrational mode } \\ w & \text { value at wall or surface } \\ w & \text { value at wall or surface } \\ \infty & \text { freestream value }\end{array}$

\section{INTRODUCTION}

Just prior to encountering and orbiting Jupiter, the Galileo spacecraft will release a probe which will enter the Jovian atmosphere. During the initial aerobraking phase, the 45-degree blunted-cone probe will be protected from heating by a carbon phenolic shield. Once the probe velocity has been reduced from $47.5 \mathrm{~km} / \mathrm{s}$ to $0.74 \mathrm{~km} / \mathrm{s}$, and entry heating has diminished, the probe will eject its heat shield and deploy a parachute. During descent, the probe will make several in situ measurements of atmospheric properties and transmit that data to the orbiting spacecraft. However, an Atmospheric Structure Experiment, ${ }^{1}$ similar to that employed in the Pioneer Venus mission, will also be on the Galileo probe to measure deceleration during the initial high-altitude entry phase. This experiment deduces atmospheric density, pressure, and temperature from deceleration measurements so long as the vehicle drag co- 
efficient is known a priori. The instrument is sufficiently sensitive to detect any deceleration exceeding $10^{-5} \mathrm{~m} / \mathrm{s}^{2}$. Consequently, meaningful properties can be assessed for the Jovian upper atmosphere where the probe encounters highly rarefied flow during entry just prior to peak heating and ablation of the heat shield. This flow regime is bounded by the effective free molecule limit at $750 \mathrm{~km}$ altitude $\left(\operatorname{Re}_{\infty}=0.1\right)$ and the near-continuum limit at $350 \mathrm{~km}$ $\left(\operatorname{Re}_{\infty}=1,000\right)$. Note that since Jupiter has no identifiable surface, altitude is measured relative to the 1.0 barr pressure level in the Jovian atmosphere.

Accuracy of the experiment, however, depends upon the accuracy with which the probe drag coefficient is estimated. Intrieri ${ }^{2}$ conducted a series of experiments in the ballistic range facilities at NASA Ames Research Center to measure the drag of several blunt-body configurations including the probes from Pioneer Venus and Galileo. Results for each were very similar, leading to values near $C_{\mathrm{D}}=1.1$ for $\mathrm{Re}_{\infty}>1,000$. However, $C_{\mathrm{D}}$ rises significantly (approaching values near 2.0) for decreasing $\mathrm{Re}_{\infty}$ below 1,000, although Intrieri's experiments were not reliable in the rarefied regime. Furthermore, the experimental surface materials, gas species, and flow conditions (Mach number, density, etc.) differed significantly from those anticipated for the probe entry at a given Reynolds number.

Due to the lack of sufficient experimental data, the probe aerodynamics must be estimated computationally. Unfortunately, the flow regime is ill-suited to simulations which are based upon the continuum Navier-Stokes equations due to limitations in the constitutive relations for heat flux and shear stress. Instead, highly rarefied flows, for which the ratio of molecular mean free path to a body dimension is large (Knudsen number, $K n>0.10$ ), are best simulated computationally with direct simulation Monte Carlo (DSMC) particle methods. ${ }^{3,4}$ Here, gas dynamics is modeled directly by the motion and interaction of thousands or millions of discrete particles. Particles which strike the vehicle may reflect back into the flow with velocities and internal energies corresponding to full or partial accommodation to the surface. The simulation permits accurate assessment of vehicle aerodynamics and heating along with properties of the flow field.

The objective of the present study was to assess the drag coefficient for the Galileo probe during entry from $750 \mathrm{~km}$ down to $350 \mathrm{~km}$ altitude in the Jovian atmosphere. Due to uncertainties in the applicable surface thermal accommodation coefficient $A$, simulations were repeated using different values to quantify its effects upon the vehicle entry. Surface heating was evaluated to determine the extent of pyrolysis of the heat shield through the use of the Charring Material Thermal Response and Ablation (CMA) program. This code models transient convective heating, radiation, in-depth conduction, heat capacity, and the flow of pyroly- sis gases through the porous material. Together, these codes simulate the entry environment and response of the Galileo probe to estimate the appropriate vehicle aerodynamics required for this and other aerobraking missions.

\section{SIMULATION MODELS}

The DSMC code employed in the present study was developed by Baganoff and McDonald ${ }^{4,5}$ and enhanced for better application to rarefied aeropass maneuvers. ${ }^{6}$ The flow field is divided into cubic cartesian cells to facilitate selection of colliding particles and sampling of macroscopic flow properties. The body geometry is modeled by a composite of planar facets in those cells through which the body surfaces pass. Each surface facet collects statistics regarding momentum and energy flux and may assume a surface temperature independent of neighboring facets. To simulate entry with the DSMC method, one must first specify properties of both gas-gas and gas-surface interactions.

\section{Gas-Gas Interaction Models}

Molecular interaction is simulated by the Variable HardSphere (VHS) model of Bird ${ }^{7}$ in which the collision outcome corresponds to isotropic scattering, akin to the mechanics of hard sphere interactions. The collision rate, however, corresponds to an inverse power-law intermolecular potential of exponent $\alpha$. This parameter must be specified between the limits of the Maxwell molecule $(\alpha=4)$ and the Hard sphere $(\alpha=\infty)$, and may be estimated from the known temperature dependence of gas viscosity $\mu$ as follows,

$$
\frac{\mu}{\mu_{\mathrm{ref}}}=\left(\frac{T}{T_{\mathrm{ref}}}\right)^{\frac{1}{2}+\frac{2}{\alpha}}
$$

Transport properties of the Jovian atmosphere, thought to be composed by a mixture of $89 \% \mathrm{H}_{2}$ and $11 \% \mathrm{He}$, were calculated from kinetic theory by Biolsi. ${ }^{8}$ That work employed sophisticated semiempirical interaction potentials to solve the detailed collision integrals for viscosity, thermal conductivity, and binary diffusion for the gas mixture. $\mathrm{Bi}$ olsi's results for viscosity are plotted in Fig. 1 and compared to curves corresponding to the VHS model. The VHS parameters which yield best agreement in the figure and were employed in the current work are given by $\mu_{\text {ref }}=2.24 \times 10^{-5} \mathrm{~kg} / \mathrm{m} \cdot \mathrm{s}, T_{\text {ref }}=1000 \mathrm{~K}$, and $\alpha=10.5$.

Of additional concem in gas-gas interactions is the inelastic exchange of molecular energies due to relaxation of the internal energy modes for rotation and vibration. The mechanics of these exchange processes are modeled in the DSMC code by the methods of Borgnakke and Larsen ${ }^{9}$ and Haas, et al. ${ }^{10}$ These involve partitioning post-collision thermal energies in a manner which corresponds to equilibrium 
distributions. The rates of rotational and vibrational relaxation are dictated by collision numbers $Z_{r}$ and $Z_{v}$, respectively. Solutions of the master equation using state-tostate transition probabilities estimated from quasi-classical methods ${ }^{11-13}$ have led to rotational relaxation rates in $\mathrm{H}_{2}$ described by $Z_{r}=100$. Simple application of the Millikan and White ${ }^{14}$ empirical expression for vibrational relaxation rates suggests that $Z_{v}$ is several orders of magnitude larger than $Z_{r}$, leading to exceedingly slow vibrational excitation. Furthermore, given the high characteristic temperature of vibration $\left(\theta_{v}=6320 \mathrm{~K}\right)^{11}$ for $\mathrm{H}_{2}$, it is unlikely that the vibrational mode will contribute significantly to the thermophysics of this flow and was therefore neglected in the present work.

DSMC methods typically model molecular rotation as a continuous energy mode with two degrees of freedom, $\zeta_{r}=$ 2. However, at freestream conditions, the quantized nature of the rotational mode for $\mathrm{H}_{2}$ leads to an effective number of degrees of freedom below two as a result of its high characteristic temperature $\left(\theta_{r}=85.33 \mathrm{~K}\right) .{ }^{15}$ As plotted in Fig. 2, dependence of $\zeta_{r}$ upon temperanure $T$ is described by the rotational partition function and may be expressed as follows,

$$
\zeta_{r}=2 \frac{\sum_{j} g_{j} r_{j} \exp \left(-r_{j}\right)}{\sum_{j} g_{j} \exp \left(-r_{j}\right)}
$$

where the normalized energy $r_{j}$ and degeneracy $g_{j}$ per rotational quantum level $j$ are defined as follows, ${ }^{16}$

$$
\begin{aligned}
& r_{j}=j(j+1) \frac{\theta_{r}}{k T}, \\
& g_{j}=2 j+1 .
\end{aligned}
$$

Rather than encorporate a detailed model of the quantized rotational mode into the code, a simpler adaptation of the continuous energy model was employed in the present work. First, it was recognized that collisions promoting rotational excitation will occur predominantly in the hightemperature regions of the flow. Since the rotational degrees of freedom would become fully excited in these regions, the normal Borgnakke-Larsen model for exchange mechanics was employed assuming $\zeta_{r}=2$. However, since the freestream temperature was fairly low, incoming particles were initialized such that the mean rotational energy was given by $\zeta_{r} k T / 2$ where $\zeta_{r}<2$ is evaluated from Eq. (2). This prevents the freestream from being too energetic in rotation, such as would occur if initialized with $\zeta_{r}=2$.

\section{Gas-Surface Interaction Models}

Details regarding the interaction of gas molecules with surfaces are not well understood in general, and are dependent upon several factors including surface roughness, impact dynamics, molecular potentials, and thermal energies. However, simple engineering models often suffice to simulate interaction phenomena. In the present work, a single thermal accommodation coefficient $A$ describes the fraction of particles which accommodate fully to the surface versus those which reflect specularly. Full accommodation implies diffuse reflection of the particle from the surface with thermal energy corresponding to the surface wall temperature, $T_{w}$. Rather than assign some temperature to the surface, the code couples a simple surface heat transfer model into the flow solution to compute $T_{w}$ directly. ${ }^{17,18}$ This model assumes that each surface facet is in radiative equilibrium with space at temperature $T_{\mathrm{D}}=150 \mathrm{~K}$, leading to the energy balance given by

$$
q-\varepsilon \sigma\left(T_{w}^{4}-T_{D}^{4}\right)=0 .
$$

Here, $q$ is the net convected heat flux to each facet accounting for both incident and reflected energy. As will be described later, this model can be enhanced to account for material heat capacity, thermal conductivity through the heat shield, and pyrolysis of the surface material.

Definitive values for $A$ and $\varepsilon$ for the interaction of Jovian atmospheric gases upon carbon phenolic material at flight conditions are not readily available. As used in the this study, a surface emissivity of $\varepsilon=0.85$ was suggested by Bueche $^{19}$ from ground-based experiments, flight data, and theoretical predictions, and is at least consistent with estimates cited elsewhere. ${ }^{20-23}$ Appropriate values for $A$ may be found with even less certainty than $\varepsilon$ from limited related experimental data ${ }^{24}$ and theoretical ${ }^{25}$ results. It therefore proved necessary to repeat simulations using different values of $A$ to assess sensitivity of the results to this parameter.

\section{SIMULATION RESULTS}

The DSMC code was used to simulated entry of the Galileo probe at several points along its trajectory from $735 \mathrm{~km}$ altitude to $353 \mathrm{~km}$. Table 1 lists the simulation conditions for each case with atmospheric data taken from Orton. Note that time is measured relative to the $735 \mathrm{~km}$ trajectory point. For all cases the velocity was assumed to be $47,450 \mathrm{~m} / \mathrm{s}$ and the Knudsen number and Reynolds number were based on the probe diameter $(1.265 \mathrm{~m})$. The grid resolution employed in the present work is defined in Table 1 by the size of the probe diameter measured in cell-lengths, and was sufficiently fine to yield less than $1 \%$ error in drag and heating. ${ }^{26}$ The geometry of the probe is compared to the simulation models in Fig. 3. Note that only one quadrant of 
the probe was simulated, taking advantage of two planes of symmetry of the body.

In general, flows with greater $\mathrm{Re}_{\infty}$ require greater resolution in order to resolve flow gradients and avoid overpredicting drag and heat transfer. However, the required size of the flow domain increases with lower $\mathrm{Re}_{\infty}$ because molecules which reflected from the probe surface are capable of diffusing far into the flow when collisions are scarce. The extent of the upstream diffusion of particles is depicted in the plot of flow temperature along the stagnation streamline in Fig. 4. The upstream domain boundary for each simulation case was sufficiently far upstream that the translational temperature was near its freestream value to prevent overprediction of heat transfer and drag. ${ }^{26}$ Density profiles along the stagnation streamline are plotted in Fig. 5 and also exhibit the effects of rarefaction. Note that no clear shock structure is observed since the shock is fully merged with the body layer. Density rose considerably near the body surface due to particle reflection from the relatively cold surface.

For each case, the simulation employed at least 16 particles per cell in the freestream and roughly 3 to 7 million particles total. The code was optimized for vectorprocessing on Cray supercomputers, requiring roughly $0.6 \mu \mathrm{sec} /$ particle/timestep on the Cray C-90 or a total run time of 5,000-8,000 CPU seconds depending upon the case. Memory requirements ranged from 50 to 200 megawords.

Results of each case, identified by $\mathrm{Re}_{\infty}$, are presented in Table 2. The cases were run with $A=0.75$ and several were repeated with $A=\{0.5,0.9\}$. Heating of the nose region was highly sensitive to accommodation coefficient $A$ while drag was fairly insensitive, particularly for the highest and lowest $\operatorname{Re}_{\infty}$ cases. Simulated drag coefficients for the Galileo probe are plotted in Fig. 6 and compared to the experimental ballistic range results of Intrieri for spheres and the Pioneer Venus probe. Unfortunately, Intrieri's results for the Galileo probe were all at high angles of attack and were of questionable quality at low $\operatorname{Re}_{\infty}$.

In general, drag dropped with increasing $\mathrm{Re}_{\infty}$ above 10 , but did not appear to blend well with the high-Re $e_{\infty}$ experimental data. However, the experiments were performed at lower Mach numbers (roughly $\mathrm{M}_{\infty}=14.5$ ) in $\mathrm{CO}_{2}$ compared with the high Mach numbers expected for Galileo entry in the $\mathrm{H}_{2}$-He Jovian atmosphere. Indeed, for comparing highly rarefied flows, a suitable parameter for correlating the data would be the Knudsen number $\mathrm{K} n_{\infty}$. Re-plotting the data in Fig. 7 suggests a smoother transition between the simulation results and the experimental work.

Nonetheless, it was instructive to simulate a few of Intrieri's experiments for more direct comparison. Spheres flying at roughly $\mathrm{M}_{\infty}=14.5$ in pure $\mathrm{CO}_{2}$ for $\mathrm{Re}_{\infty}=$ $\{192,4564\}$ were simulated with the DSMC code employing the same surface description as above. The results are included in the drag plots and appear to follow the experimental trends quite well. Additional simulations will be performed to reproduce the Pioneer Venus probe experiments and results will be reported in the final paper.

\section{Surface Temperatures and Pyrolysis}

The DSMC calculations above employed a radiativeequilibrium energy balance to compute the surface temperature. This boundary condition neglects any effects of heat capacitance and heat conduction in the spacecraft heat shield and, therefore, provides an upper bound for the surface temperature. The calculations also neglected pyrolysis from the heat shield which can be significant for a highenergy incident flow at very low densities.

To check the validity and accuracy of these assumptions, the CMA code ${ }^{27,28}$ was used to calculate the onedimensional heat transfer into the carbon phenolic heat shield at the stagnation point. Temperature-dependent material properties, surface re-radiation, and in-depth pyrolysis were included in the calculation, but surface ablation was neglected. The initial temperature was estimated to be $150 \mathrm{~K}$ just prior to entry from deep space. The aerothermal heat flux was taken from the DSMC results associated with $A=0.75$ appearing in Table 2 .

Figure 8 presents the transient surface temperature at the stagnation point during Galileo entry. As expected, the CMA-calculated surface temperature is always below the radiative-equilibrium temperature. The temperature difference between the two results increases from about $200 \mathrm{~K}$ at the initial time $\left(\operatorname{Re}_{\infty}=0.098\right)$ to $1130 \mathrm{~K}$ at 55 seconds $\left(\operatorname{Re}_{\infty}=926\right)$.

Figure 9 compares the stagnation point pyrolysis-gas mass flux with the free stream mass flux. The pyrolysis gas flux becomes significant between 43 and 48 seconds as the material temperature rises from $600 \mathrm{~K}$ to $1000 \mathrm{~K}$. At 46.4 seconds ( $R e=103.4$ ) the pyrolysis gas flux is more than 20 times the free stream mass flux.

Perhaps the drag coefficient does not depend strongly on the surface temperature which can itself be significantly below the radiative equilibrium value during entry. However, the surface mass flux due to pyrolysis of the material is significant once the probe drops below roughly $420 \mathrm{~km}$. This could lead to a noticeable increase in drag and a decrease in heating. Thorough simulation requires that this mass flux be coupled directly into the DSMC code. Such modifications are underway and the results will be reported in the final paper.

\section{Concluding Remarks}

Drag on the Galileo probe during initial entry into the Jovian atmosphere was computed with a DSMC method, and the results were consistent with the experimental re- 
sults. However, drag varies considerably with Reynold's numbers in the range $10<\operatorname{Re}_{\infty}<10,000$ at the high $\mathrm{M}_{\infty}$ flight conditions anticipated for the probe. Results correlate better with experiment when plotted against Knudsen number rather than Reynolds number under highly rarefied conditions. Simple models for gas-gas and gas-surface interaction permit efficient yet meaningful simulation of the flow, although the effects of significant surface pyrolysis warrant further investigation. Uncertainties in the thermal accommodation coefficient $A$ have minimal impact on final results due to insensitivity of drag to $A$ despite great dependence of heating upon $A$. Further comparisons to available experimental data will be made and the pyrolysis models will be coupled directly into the DSMC code before the final paper is presented.

\section{Acknowledgements}

The authors acknowledge and appreciate the support of NASA-Ames Research Center and the Numerical Aerodynamic Simulation for use of their facilities. This work was sponsored in part (for BLH) by NASA grant NCC2-582.

\section{References}

${ }^{1}$ SeIfF, A., Kirk, D.B., Young, R.E., BlanCHARD, R.C., FIndlay, J.T. Kelly, G.M., AND SOMMER, S.C., "Measurements of Thermal Structure and Thermal Contrasts in the Atmosphere of Venus and Related Dynamical Observations: Results from the Four Pioneer Venus Probes," Journal of Geophysical Research, Vol. 85, No. A13, 1980, pp. 7903-7933. 2 Intrieri, P.F. ANd Kirk, D.B., "High-Speed Aerodynamics of Several Blunt-Cone Configurations," Journal of Spacecroft, Vol. 24, No. 2, 1987, pp. 127132.

${ }^{3}$ Bird, G.A., Molecular Gas Dynamics, Clarendon Press, Oxford, 1976.

4 Baganoff, D. And MCDonald, J.D., "A Collision-Selection Rule for a Particle Simulation Method Suited to Vector Computers," Physics of Fluids A, Vol. 2, No. 7, 1990, pp. 1248-1259.

5 MCDonalD, J.D., "A Computationally Effecient Particle Simulation Method Suited To Vector Computer Architectures," Ph.D. Thesis, Stanford University, 1989. ${ }^{6}$ HaAs, B.L., "Particle Simulation of Rarefied Aeropass Maneuvers of the Magellan Spacecraft," AIAA Paper No. 92-2923, Nashville, July 1992. To appear in $J$. Spacecraft and Rockets.

7 BIRD, G.A., "Monte-Carlo Simulation In An Engineering Context," Rarefied Gas Dynamics, at 12 th International Symposium on Rarefied Gas Dynamics, Charlottesville, VA, pp. 239-255, July, 1980.
${ }^{8}$ BIolsi, L., "Transport Properties in the Jovian Atmosphere," Journal of Geophysical Research, Vol. 83, No. A3, 1978, pp. 1125-1131.

9 BorgnakKe, C. and Larsen, P.S., "Statistical Collision Model for Monte Carlo Simulation of Polyatomic Gas Mixture," Journal of Computational Physics, Vol. 18, No. $i, 1975$, pp. 405-420.

${ }^{10}$ HaAs, B.L., McDonald, J.D., and Dagum, L., "Models of Thermal Relaxation Mechanics for Particle Simulation Methods," Journal of Computational Physics, Vol. 107, No. 2, 1993, pp. 348-358.

11 Sharma, S.P. and Schwenke, D.W., "Rate Parameters for Coupled Rotation-Vibration-Dissociation Phenomena in $\mathrm{H}_{2}$," Journal of Thermophysics and Heat Transfer, Vol. 5, No. 4, 1991, pp. 469-480.

12 Sharma, S.P., "Rotational Relaxation of Molecular Hydrogen at Moderate Temperatures," AIAA Paper No. 92-2854, Nashville, July 1992.

13 Willauer, D.L. and Varghese, P.L., "Direct Simulation of Rotational Relaxation Using State-to-State Cross Sections," Journal of Thermophysics and Heat Transfer, Vol. 7, No. 1, 1993, pp. 49-54.

14 Millikan, R.C. AND White, D.R., "Systematics of Vibrational Relaxation," Journal of Chemical Physics, Vol. 39, No. 12, 1963, pp. 3209-3213.

15 Chase, M.W. JR., Davies, C.A., Downey, J.R.JR., Frurip, D.J. MCDonald, R.A., AND SYverud, A.N., "JANAF Thermochemical Tables," Third Edition, Part I, AI-Co, Journal of Physical and Chemical Reference Data, Vol. 14, Supplement No. 1, 1985.

16 Vincenti, W.G. AND KRUger, C.H. JR., Introduction to Physical Gas Dynamics, John Wiley and Sons, Inc., New York, 1965.

17 HAAS, B.L., "Particle Simulation of Satellite Aerobraking with Couple Surface Heat Transfer," presented at the 18th International Symposium on Rarefied Gas Dynamics, Vancouver, B.C., July, 1992. To appear in published proceedings.

${ }^{18}$ HaAs, B.L., "Models for Dynamic Surface Temperatures During Rarefied Aeropass Maneuvers," AIAA Paper No. 93-2765, Orlando, July 1993. Submitted to Journal of Thermophysics and Heat Transfer.

19 BUECHE, J.F., "Effects of Improvements and Uncertainties in Thermophysical Properties on Carbon Phenolic Heatshield Thermal Performance Preditions," AIAA Paper No. 77-37290, Albuquerque, June 1977.

20 POPE, R.B., "Measurements of the Total Surface Emittance of Charring Ablators," AIAA Journal, Vol. 5, No. 12,1967 , pp. 2285-2286.

${ }^{21}$ Peterson, D.L. AND NicoletT, W.E., "Heat Shielding for Venus Entry Probes," Journal of Spacecraft, Vol. 11, No. 6, 1974, pp. 382-387. 
22 PitTs, W.C. ANd Wakefield, R.M., "Performance of Entry Heat Shields on Pioneer Venus Probes," Journal of Geophysical Research, Vol. 85, No. A13, 1980, pp. 8333-8337.

${ }^{23}$ Green, M.J. and Davy, W.C., "Galileo Probe Forebody Thermal Protection," AIAA Paper No. 811073, Palo Alto, June 1981.

24 SaXena, S.C. AND Joshi, R.K., "Thermal Accommodation and Adsorption Cœfficients of Gases," McGraw-Hill/CINDAS Data Series on Material Properties, Vol. II-1, edited by Y.S. Touloukian and C.Y. Ho, McGraw-Hill Book Co., New York, 1981.

${ }^{25}$ Hollenbeck, D. ANd Salpeter, E.E., "Surface Adsorption of Light Gas Atoms," Journal of Chemical Physics, Vol. 53, No. 1, 1980, pp. 79-86.

26 HAAS, B.L., "Flow Resolution and Domain of Influence in Rarefied Hypersonic Blunt-body Flows," AIAA Paper No. 93-2806, Orlando, July 1993. Submitted to Journal of Thermophysics and Heat Transfer.

${ }^{27}$ MOYER, C.B. AND Rindal, R.A., "An Analysis of the Chemically Reacting Boundary Layer and Charring Ablator. Part II: Finite Difference Solution for the Indepth Response of Charring Materials Considering SurTable 2: Galileo Probe Entry Simulation Results

\begin{tabular}{rccrr}
\hline \multicolumn{1}{c}{$\mathrm{Re}$} & $\mathrm{A}$ & $\mathrm{CD}$ & $\begin{array}{c}\text { q-nose } \\
(\mathrm{W} / \mathrm{m} 2)\end{array}$ & $\begin{array}{c}\text { T-nose } \\
(\mathrm{K})\end{array}$ \\
\hline 0.10 & 0.75 & 2.060 & 766 & 358.0 \\
1.03 & 0.75 & 2.048 & 6,495 & 602.4 \\
8.69 & 0.75 & 2.024 & 48,728 & 998.1 \\
34.01 & 0.75 & 1.970 & 168,523 & $1,365.1$ \\
103.41 & 0.75 & 1.896 & 448,881 & $1,737.4$ \\
322.47 & 0.75 & 1.777 & $1,201,262$ & $2,236.4$ \\
926.15 & 0.75 & 1.629 & $2,980,082$ & $2,798.3$ \\
\hline 0.10 & 0.50 & 2.061 & 474 & 318.8 \\
8.69 & 0.50 & 2.046 & 32,564 & 909.9 \\
103.41 & 0.50 & 1.934 & 309,602 & $1,580.3$ \\
926.15 & 0.50 & 1.628 & 2.085 .700 & $2,545.3$ \\
\hline 0.10 & 0.90 & 2.043 & 848 & 366.8 \\
8.69 & 0.90 & 2.008 & 57,941 & $1,050.3$ \\
103.41 & 0.90 & 1.864 & 528,677 & $1,826.8$ \\
926.15 & 0.90 & 1.648 & 3.559 .079 & $2,931.1$ \\
\hline \hline
\end{tabular}
face Chemical and Energy Balances," NASA CR-1061, June, 1968.

28 ANon., "User's Manual Aerotherm Charring Material Thermal Response and Ablation Program CMA87," Report UM-87-11/ATD, Acurex Corp., Mountain View, CA, Aug., 1987.

Table 1: Galileo Probe Entry Simulation Conditions

\begin{tabular}{ccccccccc}
\hline $\mathrm{t}$ & \multicolumn{1}{c}{ Alt. } & $\mathrm{Re}$ & $\mathrm{M}$ & $\mathrm{Kn}$ & $\mathrm{T}$ & $\begin{array}{c}\rho \\
(\mathrm{kg} / \mathrm{m} 3)\end{array}$ & $\begin{array}{c}\mathrm{D} \\
(\mathrm{cells})\end{array}$ \\
\hline \hline 0.00 & 735.00 & 0.10 & 31.47 & 415.59 & 425.0 & $2.024 \mathrm{E}-11$ & 16 \\
19.00 & 604.00 & 1.03 & 34.15 & 43.01 & 360.5 & $1.896 \mathrm{E}-10$ & 16 \\
33.50 & 506.00 & 8.69 & 37.20 & 5.55 & 303.2 & $1.422 \mathrm{E}-09$ & 16 \\
41.00 & 453.00 & 34.01 & 39.61 & 1.51 & 267.0 & $5.100 \mathrm{E}-09$ & 16 \\
46.40 & 416.00 & 103.41 & 41.86 & 0.53 & 238.7 & $1.435 \mathrm{E}-08$ & 32 \\
51.25 & 382.00 & 322.47 & 44.58 & 0.18 & 210.0 & $4.097 \mathrm{E}-08$ & 32 \\
55.50 & 353.00 & 926.15 & 46.35 & 0.07 & 194.0 & $1.114 \mathrm{E}-07$ & 48 \\
\hline \hline
\end{tabular}




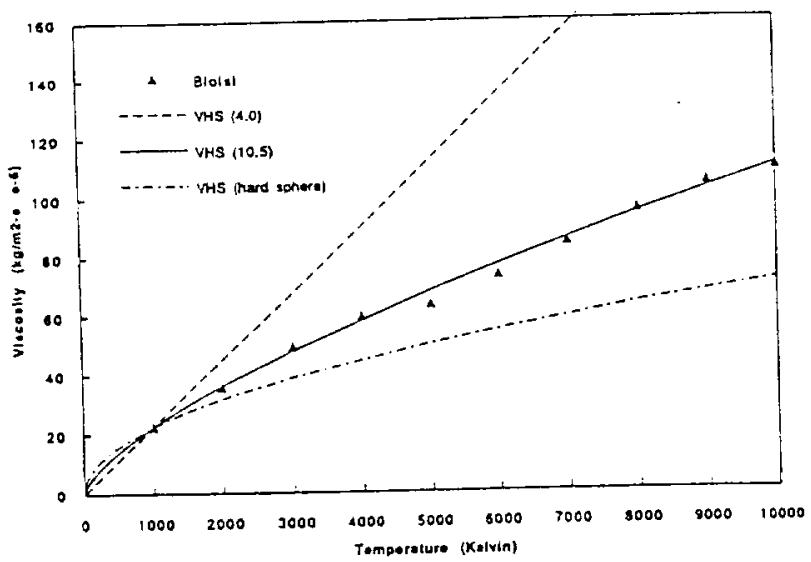

Fig. 1 Temperature variation of viscosity of the Jovian atmospheric gas mixture computed by Biolsi and fit with the VHS model.

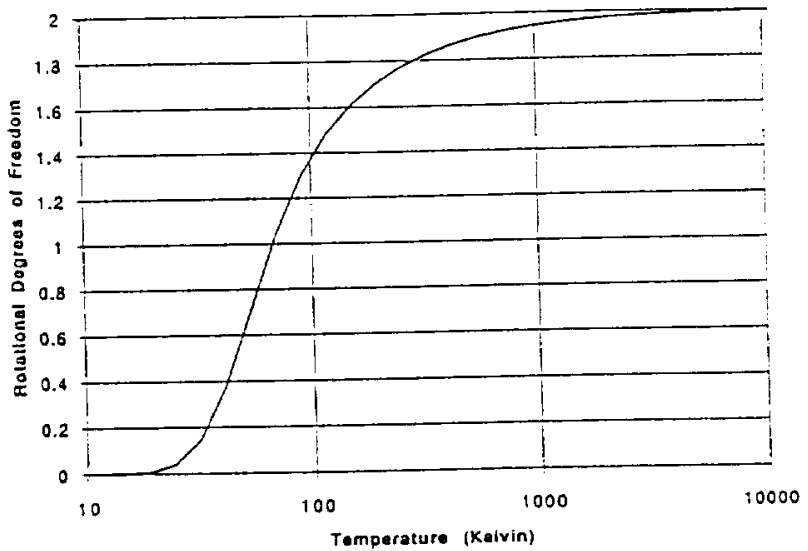

Fig. 2 Temperature dependence of the rotational degrees of freedom for $\mathrm{H}_{2}$.

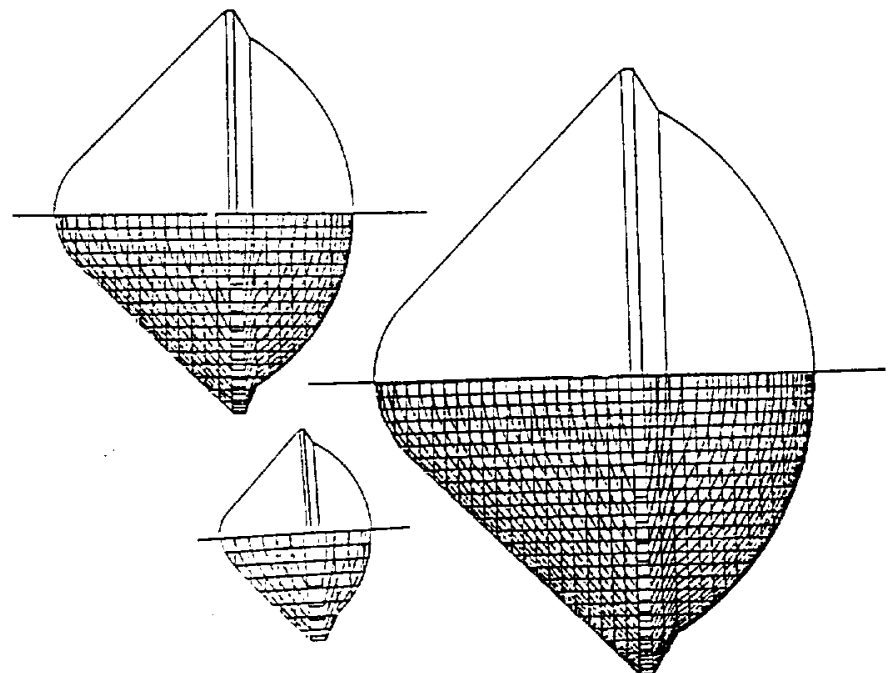

Flg. 3 Comparisons of probe geometry to simulation configurations employing differing resolutions.

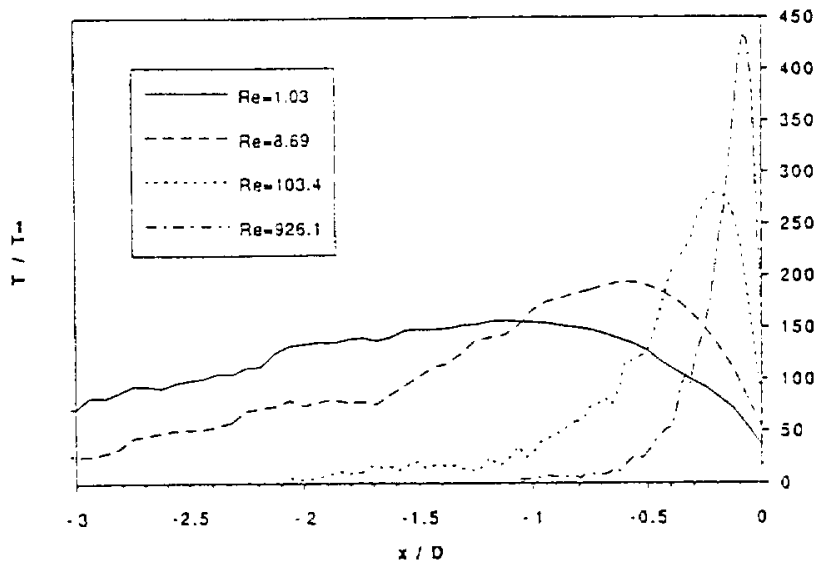

Fig. 4 Translational temperature along the stagnation streamline ahead of the probe for entry at several values of $\operatorname{Re}_{\infty}$.

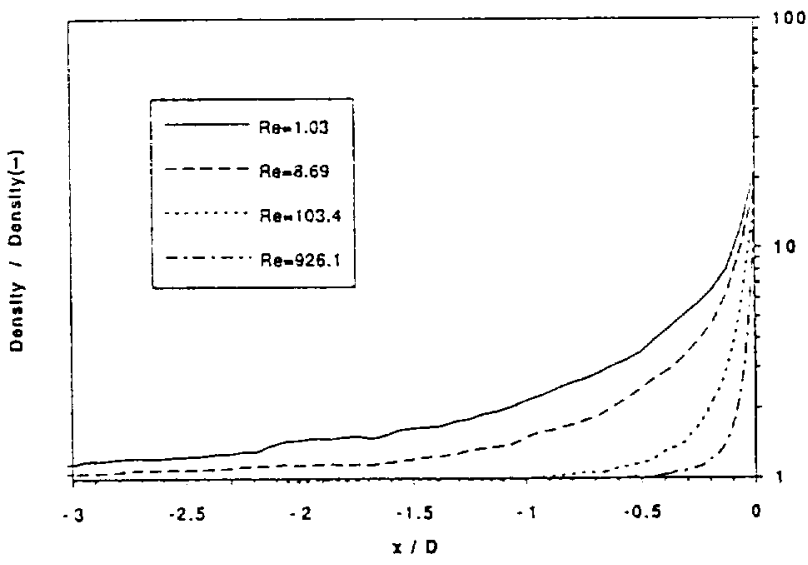

Fig. 5 Mass density along the stagnation streamline ahead of the probe for entry at several values of $\operatorname{Re}_{\infty}$.

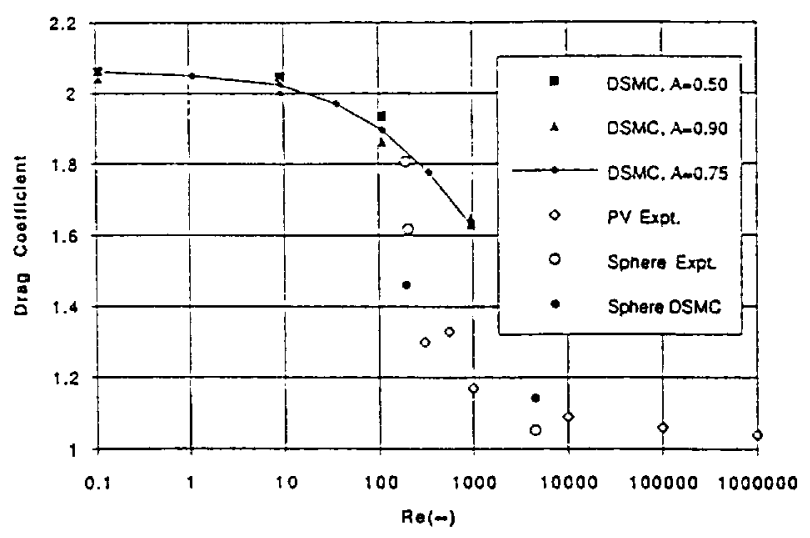

Fig. 6 Drag coefficients from DSMC simulation of Galileo enry compared to results of related experiments of Intrieri; includes results from simulations of sphere experiments. Results ploted against $\operatorname{Re}_{\infty}$. 


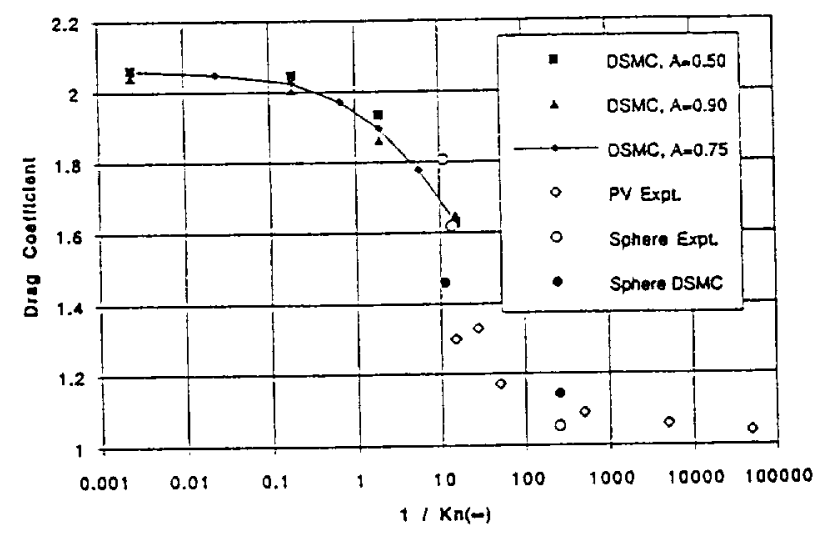

Fig. 7 Drag coefficients from DSMC simulation of Galileo entry compared to results of related experiments of Intrieri; includes results from simulations of sphere experiments. Results plotted against $\mathrm{K} \mathrm{n}_{\infty}$.

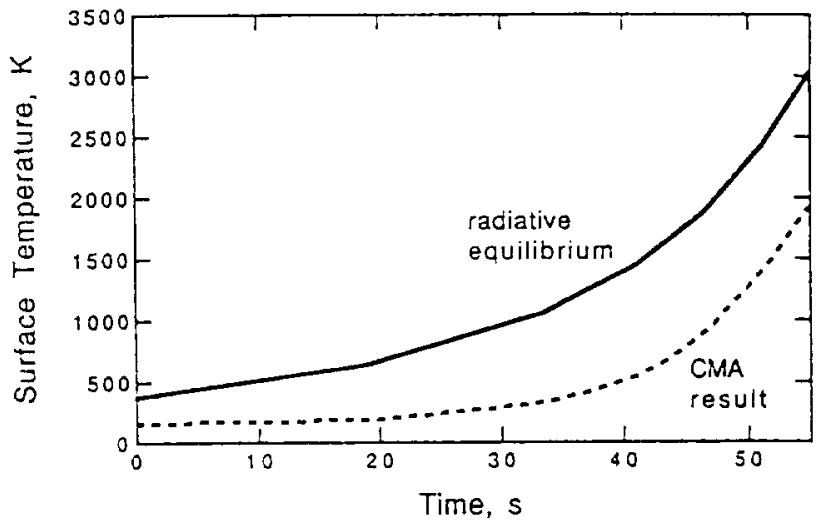

Fig. 8 Radiative-equilibrium and CMA-calculated stagnation point material temperature for initial entry period of the Galileo probe.

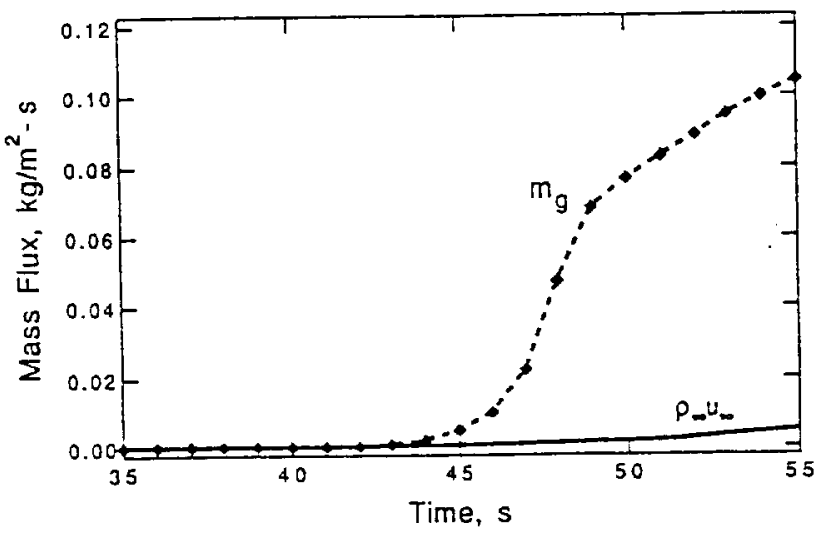

Fig. 9 Comparison of freestream mass fiux and CMA-calculated pyrolysis gas mass efflux from the probe surface during inital entry phase. 